\title{
THE DYNAMIC ANALYSIS OF CONTINUOUS-TIME LIFE-CYCLE SAVINGS GROWTH MODELS*
}

\author{
John LAITNER \\ University of Michigan, Ann Arbor, MI 48109, USA
}

Received December 1985, final version January 1987

\begin{abstract}
Working with the framework of a continuous-time overlapping-generations model, this paper examines equilibrium growth paths (consistent with perfect foresight). In particular, we develop a methodology for characterizing the adjustment paths converging to new stationary states following exogenous shocks. The solution technique yields sequences of derivatives approximating the true dynamic paths and bounds for the errors of approximation. In practice, aggregating behavior over discrete-time intervals can save a great deal of effort - considerably enhancing the set of models feasible for analysis; our error bounds enable us to aggregate without compromising our results to an unknown degree.
\end{abstract}

\section{Introduction}

This paper attempts to develop a methodology for investigating the dynamic behavior of continuous-time decentralized growth models composed of overlapping generations of finite-lived families. The households differ from one another (at minimum) in terms of birth dates, and each engages in private life-cycle utility maximization. Numerous studies have employed the basic framework - see, for instance, Tobin (1967) on national wealth accumulation, Summers (1981) on taxation, Kotlikoff (1979) on social security, Laitner (1982) on portfolio implications of monopoly, and Arthur and McNicoll (1978) on population.

Although the investigations of Tobin and others focus on stationary-state growth paths and on comparisons of the different stationary solutions corresponding to different parameter values, transition paths between such states are also potentially very important. In particular, since experience with models related to those listed above - see Laitner (1984) and Auerbach and Kotlikoff (1984) - suggests that fairly long adjustment periods follow many types of exogenous shocks, a study of the effects of a natural or policy change over, say, the change's first decade will generally require a dynamic analysis.

In turning our attention to dynamic time paths, we limit ourselves to those which represent sequences of equilibrium states - consistent with perfect

*This research was supported by the National Science Foundation, Grant SES-8106555. 
foresight on the part of all agents. Steady states represent, of course, a theoretically and empirically interesting subset of such paths. All of our analyses start from and (asymptotically) converge to stationary solutions.

Overlapping-generations models tend to be complicated to study, and difficulties increase as we depart from stationary solutions. For their dynamic analysis, therefore, Auerbach and Kotlikoff (1984) employ a numerical procedure: they choose a long time interval (following an exogenous shock) over which they assume the transition will fully run its course, they select a grid over the interval, and they use an analogue of Newton's method to find a vector consisting of state variables at each grid-point time and satisfying the equilibrium conditions of the model. Drawbacks are that such an approach reveals little about the saddlepoint nature of the dynamic system and related questions of uniqueness. Furthermore, although the interests of computational tractability favor a coarse grid, the Auerbach-Kotlikoff technique provides no information on the errors of approximation different choices might imply.

Laitner (1984) suggests generating a sequence of derivatives, the sequences defining transitions following an infinitesimal shock. ${ }^{1}$ An intermediate step computes an eigenvalue vector which fully characterizes the underlying phase diagram. On the other hand, since the approach is designed for discrete-time models, limitations are a lack of strict compatibility with papers employing continuous time and the seeming arbitrariness of any given choice of a period length. Also, dimensionality is again an issue: if households live a maximum of $T$ years and our time unit is a year, Laitner's dynamic analysis involves a vector equation roughly of order $2 T$. If we want quarterly results, vector lengths increase fourfold; if we want to use time units longer than one year to reduce computational burdens (and, perhaps more critically, computer round off errors), approximation errors, which (again) are unknown, may be severe.

This paper works directly with a continuous-time model. We characterize transition paths with time-dependent derivatives. Thus, although our model's equilibrium condition is highly non-linear, the dynamic analysis depends on a linear equation. Unfortunately, the latter is an integral equation. Sections 3 and 4 below develop a method of coping with it. The procedure involves setting up a grid. After solving the resulting discrete-time system, however, we can easily compute dynamic 'multipliers' for interim times. We do obtain an eigenvalue characterization of our model's saddlepoint structure. Most importantly, our solution yields upper bounds for the approximation errors (for all multipliers) induced in our discretization steps. These bounds potentially allow us to work with coarser grids than we would otherwise trust - and this possibility is borne out in the numerical examples of section 6 . The coarser grids, in turn, suggest that elaborations to the underlying economic model, such as incorporating multiple capital stocks, are feasible in practical problems.

\footnotetext{
${ }^{1}$ The derivatives are analogous to familiar comparative stationary-state multipliers.
} 


\section{Dynamic multipliers}

This section first illustrates our method of presenting dynamic results, with multipliers which are functions of time, in the context of a very simple model. Then it sets up our life-cycle savings growth framework. Finally, it constructs our equation defining multipliers for the life-cycle system.

\subsection{A simple example}

To illustrate our procedure for investigating non-stationary growth paths, consider for a moment the familiar Solow (1956) model with a constant average propensity to save. If $k_{t}$ is the capital-to-'effective' labor ratio and $\theta$ is any parameter, the model has an equilibrium condition of the form

$$
\dot{k}_{t}=\phi\left(k_{t}, \theta\right) \text {. }
$$

If the analysis begins at time 0 , with $k_{0}$ given, the equation's solution, say, $\psi(\cdot)$, has the form

$$
k_{t}=\psi\left(t, k_{0}, \theta\right), \quad \text { all } t \geq 0 .
$$

Let there be a permanent change in $\theta$ beginning at time 0 . Let $k_{\infty}=\Psi(\theta)$ be a stationary solution [so that $0=\phi\left(k_{\infty}, \theta\right)$ ]. To compare stationary growth paths before and after time 0 , we often compute a comparative steady-state 'multiplier'

$$
\partial k_{\infty} / \partial \theta=-\left[\partial \phi\left(k_{\infty}, \theta\right) / \partial \theta\right] /\left[\partial \phi\left(k_{\infty}, \theta\right) / \partial k\right] .
$$

Proceeding in the same fashion for transition-path multipliers, differentiate (1) with respect to $\theta$. Referring to Coppel (1965, theorem 6, p. 22) to justify changing the order of differentiation on the left side and using (2),

$$
\begin{aligned}
\partial\left[\partial \psi\left(t, k_{0}, \theta\right) / \partial \theta\right] / \partial t= & {\left[\partial \phi\left(\psi\left(t, k_{0}, \theta\right), \theta\right) / \partial k\right] } \\
& \cdot\left[\partial \psi\left(t, k_{0}, \theta\right) / \partial \theta\right] \\
& +\left[\partial \phi\left(\psi\left(t, k_{0}, \theta\right), \theta\right) / \partial \theta\right]
\end{aligned}
$$

This is a first-order differential equation in $\partial \psi\left(t, k_{0}, \theta\right) / \partial \theta=\partial k_{t} / \partial \theta$. Using

$$
\partial \psi\left(0, k_{0}, \theta\right) / \partial \theta=\partial k_{0} / \partial \theta=0,
$$

we should be able to solve (4) for a sequence of dynamic multipliers,

$$
\partial k_{t} / \partial \theta=\partial \psi\left(t, k_{0}, \theta\right) / \partial \theta, \quad \text { all } t \geq 0 .
$$

Solow's model implies the economy will, in general, reside in a stationary state in the absence of a recent shock; hence, the case with $k_{0}=\Psi(\theta)$ is especially significant. $\left[k_{0}=\Psi(\theta)\right.$ is implicit in the analysis of (3).] With such an initial condition,

$$
\psi\left(t, k_{0}, \theta\right)=k_{0}=k_{\infty}, \quad \text { all } t \geq 0
$$


So, (4) becomes

$$
\begin{aligned}
\partial\left[\partial \psi\left(t, k_{\infty}, \theta\right) / \partial \theta\right] / \partial t= & {\left[\partial \phi\left(k_{\infty}, \theta\right) / \partial k\right] \cdot\left[\partial \psi\left(t, k_{\infty}, \theta\right) / \partial \theta\right] } \\
& +\left[\partial \phi\left(k_{\infty}, \theta\right) / \partial \theta\right]
\end{aligned}
$$

a linear equation (in $\partial k_{t} / \partial \theta$ ) with constant coefficients. In this situation, for a given time- 0 shock $\mathrm{d} \theta,\left(\partial k_{t} / \partial \theta\right) \cdot \mathrm{d} \theta$ measures the capital-to-labor ratio's equilibrium deviation, after $t$ periods, from its original stationary value. ${ }^{2,3}$

\subsection{Our life-cycle model}

The underlying economic model on which this paper focuses closely resembles Tobin's (1967). This subsection reviews the framework briefly. It also prepares the groundwork for the illustrative numerical example of section 5 .

Our model has a single output good, net national product, and an aggregate production function $F(\cdot)$ : if $K$, is the aggregate physical capital stock, $E$, the aggregate 'effective' labor force, $Y_{t}$ the net national product, and $\theta$ is a constant - to be thought of as 1 at this point,

$$
Y_{t}=\theta \cdot F\left(K_{t}, E_{t}\right) \text {. }
$$

We assume $F(\cdot)$ is increasing and concave in each argument and exhibits constant returns to scale. Define

$$
k_{t} \equiv K_{t} / E_{t} \quad \text { and } \quad f\left(k_{t}\right) \equiv F\left(k_{t}, 1\right)
$$

Units of $Y_{t}$ are homogeneously divisible into consumption and investment. Assuming competitive factor pricing and (constant) proportional tax rates $\tau_{w}$ and $\tau_{r}$ for wage and interest earnings, if $w_{t}$ is the time- $t$ net-of-tax wage rate and $r_{t}$ the net-of-tax interest rate,

$$
\begin{aligned}
& w_{t}=\left(1-\tau_{w}\right) \cdot \theta \cdot\left[f\left(k_{t}\right)-k_{t} \cdot f^{\prime}\left(k_{t}\right)\right] \equiv W\left(k_{t}, \theta\right), \\
& r_{t}=\left(1-\tau_{r}\right) \cdot \theta \cdot f^{\prime}\left(k_{t}\right) \equiv R\left(k_{t}, \theta\right) .
\end{aligned}
$$

In our numerical examples $F(\cdot)$ is CES. Labor's share of total income in the stationary state is $0.82, \tau_{w}=\tau_{r}=0.3$ in most cases, and the production elasticity of substitution is 0.5 in most cases. ${ }^{4}$

\footnotetext{
${ }^{2}$ If $k_{\infty}=\Psi(\theta)$ is a 'stable' stationary solution, $\lim _{t \rightarrow \infty} \partial k_{t} / \partial \theta=\partial k_{\infty} / \partial \theta$.

${ }^{3}$ Notice that this subsection is similar to a special case of Aoki (1980).

${ }^{4}$ Labor's share comes from the 1978 national accounts - with labor's share of proprietor's income set equal to labor's total share of national income. The tax proportions come from the 1978 national accounts figures for federal, state, and local tax collections less all transfer payments other than social-security retirement benefits. Below we arbitrarily try a higher tax rate on the return to capital, $\tau_{r}=0.40$, with $\tau_{w}$ adjusted to keep total collections constant. Lucas (1969) estimates production elasticities.
} 
Each household lives $T$ years. As in Tobin (1967), a household includes two adults with uncertain life spans (although mortality tables are known and invariant) and children. Institutions provide actuarially fair life insurance. For simplicity, we omit population growth; thus, every household has two children. The offspring are born and leave home at prescribed times.

Consider a household started at time $t$. Let its age now be $u$. As in Tobin, assign adult equivalency weights to children. The weights plus each adult's probability of dying after age $u$ [see Tobin (1967) and Yaari (1965)] yield a schedule $n(u), u \in[0, T]$, of 'equivalent adults' for all families. For the household under consideration, let $c(u, t)$ be (real) consumption per adult, let $a(u, t)$ be family asset holdings, let $l(u)$ give the household's labor supply in natural units (an exogenous variable in this paper), and let $l(u) \cdot \mathrm{e}^{\gamma(t+u)}$ give its 'effective' supply - the rate of labor-augmenting technical progress being $\gamma$. We posit a Bergson family utility function - allowing the existence of a stationary state for the economy even if $\gamma \neq 0$ [see Katzner (1970, theorem 2.4-4)]. ${ }^{5}$ The same Bergson parameter $\beta<1, \beta \neq 0$ applies for all households (although this last assumption is by no means crucial). A household (born at $t$ ) solves at age $s$

$$
\max _{c(u, t)} \int_{s}^{T} n(u) \cdot\left[c(u, t)^{\beta} / \beta\right] \mathrm{d} u,
$$

subject to

$$
\begin{aligned}
& \partial a(u, t) / \partial u=r_{t+u} \cdot a(u, t)+w_{t+u} \cdot \mathrm{e}^{\gamma(t+u)} \cdot l(u)-n(u) \cdot c(u, t), \\
& a(s, t) \text { given, } \quad a(T, t)=0 .
\end{aligned}
$$

There are neither bequests nor inheritances; so $a(0, t)=0$.

In our numerical examples, households start with an 18-year-old husband and wife, adults live a maximum of 70 additional years, survival probabilities for men and women (separately) come from a standard mortality table, minor children receive equivalent adult weights of 0.6 , and teenagers receive 0.8 . A family's first child is born when the parents are 22, the second when they are 25 . Each child leaves home at age 18. Many of our examples use $\beta=-1$. $^{6}$

Our examples use labor supply schedules proportional to the (labor) earnings figures in U.S. Department of Health and Human Services (1983, table 25 , p. 83). We use data from 1978 , the most recent year for which final numbers are available. We keep track of men and women separately, correcting the supply figures using our survival probabilities and average participation rates (for 1978) form U.S. Department of Labor (1980, table 4,

\footnotetext{
${ }^{5}$ If $\gamma=0$, we do not need Bergson functions. The analysis of sections 3-6 and, indeed, the basic course of this paper are largely unrelated to the special properties of this class of functions.

${ }^{6}$ The weights for children and teenagers come from Tobin (1967). Weber (1975) provides some estimates of $\beta$. (Tobin uses $\beta=0$.)
} 
p. 160). We also correct for social-security retirement income - see below. As in Summers (1981) and Kotlikoff (1979), we set $\gamma=0.02$.

The Euler equation [note that second-order conditions hold for problem (7)] implies

$$
\begin{aligned}
c(u, t)= & c(s, t) \cdot \exp \left\{[1 /(1-\beta)] \cdot \int_{s}^{u} r_{t+x} \mathrm{~d} x\right\} \\
c(s, t)= & \left\{a(s, t)+\int_{s}^{T} l(v) \cdot w_{t+v} \cdot \mathrm{e}^{\gamma(t+v)} \cdot \exp \left\{-\int_{s}^{v} r_{t+x} \mathrm{~d} x\right\} \mathrm{d} v\right\} \\
& / \int_{s}^{T} n(v) \cdot \exp \left\{[\beta /(1-\beta)] \cdot \int_{s}^{v} r_{t+x} \mathrm{~d} x\right\} \mathrm{d} v .
\end{aligned}
$$

Define

$$
\begin{aligned}
m(u-t) & \equiv \max \{u-t, 0\} \\
M(u-t) & \equiv 1 \quad \text { if } \quad u-t \geq 0 \\
& \equiv 0 \quad \text { otherwise. }
\end{aligned}
$$

Then for a household aged $u$ and born at time $t-u$,

$$
\begin{aligned}
a(u, t-u)= & \int_{m(u-t)}^{u} l(v) \cdot w_{t-u+v} \mathrm{e}^{\gamma(t-u+v)} \cdot \exp \left\{\int_{v}^{u} r_{t-u+x} \mathrm{~d} x\right\} \mathrm{d} v \\
& -\int_{m(u-t)}^{u} n(v) \cdot c(v, t-u) \cdot \exp \left\{\int_{v}^{u} r_{t-u+x} \mathrm{~d} x\right\} \mathrm{d} v \\
& +M(u-t) \cdot a(u-t, t-u) \cdot \exp \left\{\int_{u-t}^{u} r_{t-u+x} \mathrm{~d} x\right\}
\end{aligned}
$$

The equilibrium condition for the model equates capital used in production with capital owned by households. Noting that

$$
E_{t}=\mathrm{e}^{\gamma t} \cdot \int_{0}^{T} l(u) \mathrm{d} u
$$

we have

$$
k_{t}=\int_{0}^{T} a(u, t-u) \mathrm{d} u / E_{t} .
$$

For simplicity we omit government debt. Tax revenues finance government spending - which does not influence family consumption choices. We do, 
Table 1

Stationary Solutions.

\begin{tabular}{cccr}
\hline & \multicolumn{1}{c}{ Parameters $^{\mathrm{a}}$} & \multicolumn{2}{c}{ Stationary values for } \\
\cline { 3 - 4 } Example & \multicolumn{1}{c}{$K / Q$} \\
\hline 1 & $\rho=1.0, \beta=-1.0, \tau_{w}=0.30, \tau_{r}=0.30$ & 1.78 & 1.56 \\
2 & $\rho=1.0, \beta=0.0 \tau_{w}=0.30, \tau_{r}=0.30$ & 5.56 & 3.04 \\
3 & $\rho=1.0, \beta=-1.0, \tau_{w}=0.28, \tau_{r}=0.40$ & 1.54 & 1.40 \\
4 & $\rho=1.0, \beta=0.0, \tau_{w}=0.28, \tau_{r}=0.40$ & 4.55 & 2.76
\end{tabular}

${ }^{a}$ See text. $\rho$ is the CES exponent [the elasticity of substitution being $1 /(1+\rho)$ ].

however, add social-security retirement benefits back into household budgets (to replace the social-security portion of $\tau_{w}$ - see note 4 ) by augmenting the labor-supply figures of households of age 68 and over. ${ }^{7}$

To determine a stationary solution, set $s=0$ in (8)-(9) and $m=M=0$ in (10). Choose a prospective stationary $k$. Use it to calculate a time-independent $r$ and $w$ from (5)-(6). Substitute into (8)-(10) and then (12). Call the right-hand side of (12) $k^{*}$. Use Newton's method to adjust $k$ until $k^{*}=k$. Table 1 gives examples. Parameter values not explicitly given equal those explained above in the text. Notice that the units on $k$ depend on our choice of a labor-supply variable, but that the units on the capital-to-net national product ratio are years. ${ }^{8}$.

\subsection{Transition paths}

Many parameter changes, leading to adjustment paths and new stationary states, are possible. For the sake of simplicity and concreteness we focus on the following single one. Prior to time 0 , let the parameter $\theta$ multiplying $F(\cdot)$ equal 1. At time 0 , suppose an exogenous shock changes $\theta$ by $\mathrm{d} \theta$. The variation is unanticipated. As it occurs, all agents realize that it is permanent - corresponding, for example, to a permanent change in domestic productivity. ${ }^{9}$ We now derive an equation determining $\partial k_{t} / \partial \theta$ all $t \geq 0$ for our life-cycle model.

\footnotetext{
${ }^{7}$ We compute social-security retirement payments for 1978 from U.S. Department of Health and Human Services (1983, table 98, pp. 172-173). Benefits are assigned on the basis of survival probabilities. We assume all families are in the social-security system.

${ }^{8}$ Musgrave's (1982, tables 4 and 8, pp. 172-173) constant-dollar non-residential and residential private-capital figures for 1978 plus national-accounts data on inventories suggest an empirical $K / Q$ ratio of 1.95 .

${ }^{9}$ Our methodology could be extended to deal with changes anticipated $N$ years ahead of time or with changes known to be temporary.
} 
Differentiating (12),

$$
\partial k_{t} / \partial \theta=\int_{0}^{T}[\partial a(u, t-u) / \partial \theta] \mathrm{d} u / E_{t} .
$$

(Notice that $E_{t}$ is not a function of $\theta$.) The problem is to characterize $\partial a(u, t-u) / \partial \theta$. We proceed from (10).

As in the case of a comparative steady-state multiplier, assume that prior to time 0 the economy had reached a stationary equilibrium $k_{\infty}$. Thus, the effect of $\mathrm{d} \theta$ is a perturbation about the path $k_{t}=k_{\infty}$ all $t \geq 0$. Let

$$
\begin{aligned}
& r^{*} \equiv R\left(k_{\infty}, \theta\right), \\
& w^{*} \equiv W\left(k_{\infty}, \theta\right), \\
& \begin{aligned}
\partial w_{t} / \partial \theta= & \left(1-\tau_{w}\right) \cdot\left[f\left(k_{\infty}\right)-k_{\infty} \cdot f^{\prime}\left(k_{\infty}\right)\right] \\
& -\left(1-\tau_{w}\right) \cdot k_{\infty} \cdot f^{\prime \prime}\left(k_{\infty}\right) \cdot\left(\partial k_{t} / \partial \theta\right) \\
\equiv & w^{1}+w^{2} \cdot\left(\partial k_{t} / \partial \theta\right), \\
\partial r_{t} / \partial \theta= & \left(1-\tau_{r}\right) \cdot f^{\prime}\left(k_{\infty}\right)+\left(1-\tau_{r}\right) \cdot f^{\prime \prime}\left(k_{\infty}\right) \cdot\left(\partial k_{t} / \partial \theta\right) \\
\equiv & r^{1}+r^{2} \cdot\left(\partial k_{t} / \partial \theta\right) .
\end{aligned}
\end{aligned}
$$

Differentiating (10) about the stationary growth path,

$$
\begin{aligned}
\partial a(u, t-u) / \partial \theta= & \int_{m(u-t)}^{u} l(v) \cdot\left[w^{1}+w^{2} \cdot\left(\partial k_{t-u+v} / \partial \theta\right)\right] \\
& \cdot \mathrm{e}^{\gamma(t-v+v)} \cdot \mathrm{e}^{r(u-v)} \mathrm{d} v \\
& +\int_{m(u-t)}^{u} l(v) \cdot w^{*} \cdot \mathrm{e}^{\gamma(t-u+v)} \cdot \mathrm{e}^{r(u-v)} \\
& \cdot \int_{v}^{u}\left[r^{1}+r^{2} \cdot\left(\partial k_{t-u+x} / \partial \theta\right)\right] \mathrm{d} x \mathrm{~d} v \\
& +\int_{m(u-t)}^{u} n(v) \cdot c(v, t-u) \cdot \mathrm{e}^{r(u-v)} \\
& \cdot \int_{v}^{u}\left[r^{1}+r^{2} \cdot\left(\partial k_{t-u+x} / \partial \theta\right)\right] \mathrm{d} x \mathrm{~d} v \\
& +\int_{m(u-t)}^{u} n(v) \cdot(\partial c(v, t-u) / \partial \theta) \cdot \mathrm{e}^{r(u-v)} \mathrm{d} v \\
& +M(u-t) \cdot a(u-t, t-u) \cdot \mathrm{e}^{r t} \\
& \cdot \int_{u-t}^{u}\left[r^{1}+r^{2} \cdot\left(\partial k_{t-u+x} / \partial \theta\right)\right] \mathrm{d} x .
\end{aligned}
$$


Notice that $a(u-t, t-u)$, all $u \in[0, T]$, are fixed at time $0-$ in other words, they do not vary with $\theta$. Eq. (8) yields

$$
\begin{aligned}
\partial c(v, t-u) / \partial \theta= & {[\partial c(m(u-t), t-u) / \partial \theta] \cdot \mathrm{e}^{r(v-m(u-t)) /(1-\beta)} } \\
+ & c(m(u-t), t-u) \cdot[1 /(1-\beta)] \\
& \cdot \mathrm{e}^{r(v-m(u-t)) /(1-\beta)} \\
& \cdot \int_{m(u-t)}^{v}\left[r^{1}+r^{2} \cdot\left(\partial k_{t-u+x} / \partial \theta\right)\right] \mathrm{d} x
\end{aligned}
$$

Defining

$$
T I \equiv \int_{m(u-t)}^{T} n(v) \cdot \mathrm{e}^{\beta \cdot r(v-m(u-t)) /(1-\beta)} \mathrm{d} v
$$

Eq. (9) implies

$$
\begin{aligned}
& \partial c(m(u-t), t-u) / \partial \theta \\
& =-c(m(u-t), t-u) \cdot \int_{m(u-t)}^{T} n(v) \\
& \cdot \mathrm{e}^{\beta \cdot r(v-m(u-t)) /(1-\beta)} \cdot[\beta /(1-\beta)] \\
& \quad \cdot \int_{m(t-u)}^{v}\left[r^{1}+r^{2} \cdot\left(\partial k_{t-u+x} / \partial \theta\right)\right] \mathrm{d} x \mathrm{~d} v / T 1+\int_{m(u-t)}^{T} l(v) \\
& \cdot\left[w^{1}+w^{2} \cdot\left(\partial k_{t-u+v} / \partial \theta\right)\right] \cdot \mathrm{e}^{\gamma(t-u+v)} \cdot \mathrm{e}^{-r(v-m(u-t))} \mathrm{d} v / T 1 \\
& \quad-\int_{m(u-t)}^{T} l(v) \cdot w^{*} \cdot \mathrm{e}^{\gamma(t-u+v)} \cdot \mathrm{e}^{-r(v-m(u-t))} \\
& \quad \cdot \int_{m(u-t)}^{v}\left[r^{1}+r^{2} \cdot\left(\partial k_{t-u+x} / \partial \theta\right)\right] \mathrm{d} x \mathrm{~d} v / T 1 .
\end{aligned}
$$

Changing the order of integration where necessary, the derivation of $\partial a(u$, $t-u) / \partial \theta$ yields functions $G(\cdot)$ and $H(\cdot)$ such that (14) can be rewritten as

$$
\partial k_{t} / \partial \theta=\int_{m(u-t)}^{t+T} G(t, s) \cdot\left(\partial k_{s} / \partial \theta\right) \mathrm{d} s+H(t) .
$$

$G(\cdot)$ and $H(\cdot)$ depend on $k_{\infty}$, which has been suppressed as an argument because it is constant. Eq. (17) gives the linear integral equation mentioned in the introduction. Setting

$$
G(t, x)=0, \text { all } x \notin[t-T, t+T], \text { all } x<0,
$$


we can rewrite (17) as

$$
\partial k_{t} / \partial \theta=\int_{0}^{\infty} G(t, s) \cdot\left(\partial k_{s} / \partial \theta\right) \mathrm{d} s+H(t) .
$$

Notice that factor prices at the extreme beginning and ending of the lives of the oldest and youngest families existing at any time $t$ have a negligible influence on aggregate capital holdings. So,

$$
G(s, s-T)=0 \text { and } G(s, s+T)=0, \text { all } s \geq 0 .
$$

Our assumptions of homotheticity of preferences, linear homogeneity for $F(\cdot)$, and labor-augmenting technical progress imply

$$
\begin{aligned}
& G(t, s)=G(t+x, s+x) \text { and } \quad H(t)=H(t+x), \\
& \text { all } x \geq 0, t \geq T, s .
\end{aligned}
$$

For each $t \in(0, T)$, there will be some households in the current population born before time 0 . The asset holdings of such families at time 0 will reflect their prior behavior (in an economy in which $k_{t}=k_{\infty}$ and $\theta=1$ were thought to be permanent). The 'special' nature of these peoples' asset holdings prevents (20) from being valid before time $t=T$. After time $T$, all households initiated prior to $t=0$ are deceased.

Notice the flexibility inherent in the way our framework is set up: if different segments of the cohort born at $t$ have different parameters $\beta$, family compositions $n(\cdot)$, and/or labor supplies $l(\cdot)$, provided the fraction of families in each subgroup is constant across times $t$, we can simply develop (14)-(16) for each class and then form $G(\cdot)$ [in (17)] from a weighted average.

\section{An approximate solution}

To develop a practical method of solving (18), this section modifies arguments in Kantorovich and Krylov (1958, ch. II) - the latter being limited, in particular, to problems with $t$ contained in a bounded interval. Our procedure generates approximations, $z_{j}$, all $j=0, \ldots$, for $\partial k_{t} / \partial \theta$ at the points of a grid, and, subsequently, approximations $Z(t)$, all $t \geq 0$. Section 5 considers the existence and uniqueness of an exact solution; section 4 bounds our errors of approximation.

The first step is to replace the integral in (17)-(18) with a summation. Define

$$
\zeta(t) \equiv \partial k_{t} / \partial \theta
$$

We use Simpson's rule - see, for example, David and Rabinowitz (1975, 
p. 45). Select an even integer $n$ and a grid size $h$ with

$$
h=2 T / n \text {. }
$$

Define

$$
\begin{aligned}
& x_{0}(t)=t-T, \\
& x_{i}(t)=x_{0}(t)+i \cdot h, \quad \text { all } i=1, \ldots, n, \\
& A_{0}=A_{n}=h / 3, \\
& A_{i}=4 h / 3 \text { for } i \in\{1,3,5, \ldots, n-1\}, \\
& \quad=2 h / 3 \text { for } i \in\{2,4, \ldots, n-2\} .
\end{aligned}
$$

Then $[\operatorname{see}(19)]^{10}$

$$
\begin{aligned}
\int_{0}^{\infty} G(t, x) \cdot \zeta(x) \mathrm{d} x & \approx \sum_{i=0}^{n} G\left(t, x_{i}(t)\right) \cdot \zeta\left(x_{i}(t)\right) \cdot A_{i} \\
& =\sum_{i=1}^{n-1} G\left(t, x_{i}(t)\right) \cdot \zeta\left(x_{i}(t)\right) \cdot A_{i} .
\end{aligned}
$$

Let

$$
t_{j}=h \cdot j, \quad \text { all } j=0,1, \ldots
$$

Let $z_{j}$ be our approximation of $\zeta(t)$ at $t=t_{j}$. Looking at (18) and (21), we require that $z_{j}$ obey

$$
z_{j}=\sum_{i=1}^{n-1} G\left(t_{j}, x_{i}\left(t_{j}\right)\right) \cdot A_{i} \cdot z_{x_{i}\left(t_{j}\right)}+H\left(t_{j}\right), \quad \text { all } j \geq 0 .
$$

Because history fixes $k_{0}$,

$$
z_{0}=0
$$

Eqs. (22)-(23) define an infinite-dimensional system of linear (algebraic) equations, from which we ultimately hope to determine $z_{j}$ all $j$. Rearranging the system so that all $z$ terms are on the left, we can think of a matrix $Q$ times

\footnotetext{
${ }^{10}$ If we know $G(t, s)=0$, all $s<x_{i}(t)$, some $1 \leq i$ - and this will be true for many $t<T$ - we will want to use Simpson's rule on the largest possible even numbered block of $\left[x_{j}(t), x_{j+1}(t)\right]$ intervals and the trapezoidal rule elsewhere. For notational simplicity we omit this detail in the text. Our error calculations below will be affected very slightly - see Davis and Rabinowitz (1975) for trapezoidal-method error limits.
} 
a vector equaling a vector:

$$
Q \cdot\left(\begin{array}{c}
z_{0} \\
z_{1} \\
\vdots
\end{array}\right)=\left(\begin{array}{c}
H\left(t_{0}\right) \\
H\left(t_{1}\right) \\
H\left(t_{2}\right) \\
\vdots
\end{array}\right)
$$

An examination of (20) and (22)-(24) indicates that the non-zero elements of $Q$ form a band of maximum width $n-1$ about the principal diagonal. Eq. (19) reveals that the band replicates itself beginning with row $(n / 2)+1$ - in the sense that $Q_{i j}=Q_{i+1, j+1}$, all $i \geq(n / 2)+1$ - and that $H\left(t_{i}\right)$ is constant, all $i \geq(n / 2)$. Thus, from row $(n / 2)+1$ onward, (24) defines a constant-coefficient linear difference equation, of order $n-2$, for $z_{i}$.

For the moment, let us focus our attention on the latter equation. It has the form, defining $q_{j}^{*}$ and $q^{*}$ from (24),

$$
q_{0}^{*} \cdot z_{i}+\cdots+q_{n-2}^{*} \cdot z_{i+n-2}=q^{*}, \text { all } i \geq 1 .
$$

Let

$$
\begin{aligned}
& q_{j} \equiv-q_{j}^{*} / q_{n-2}^{*}, \quad \text { all } j=0, \ldots, n-3, \\
& q \equiv q^{*} / q_{n-2}^{*} .
\end{aligned}
$$

Then

$$
z_{i+n-2}=q_{0} \cdot z_{i}+\cdots+q_{n-3} \cdot z_{i+n-3}+q, \text { all } i \geq 1 .
$$

Converting to a system of first-order equations, if

$$
\begin{aligned}
& P \equiv\left(\begin{array}{ccccc}
0 & 1 & 0 & \cdots & 0 \\
\vdots & & & & \\
0 & 0 & 0 & \cdots & 1 \\
q_{0} & q_{1} & q_{2} & \cdots & q_{n-3}
\end{array}\right), \\
& P \cdot\left(\begin{array}{c}
z_{i} \\
\vdots \\
z_{i+n-3}
\end{array}\right)+\left(\begin{array}{c}
0 \\
\vdots \\
0 \\
q
\end{array}\right)=\left(\begin{array}{c}
z_{i+1} \\
\vdots \\
z_{i+n-2}
\end{array}\right), \quad \text { all } i \geq 1 .
\end{aligned}
$$

Experience indicates that $P$ may have a mixture of explosive and stable eigenvalues. For instance, Stiglitz's (1974) model with physical capital and a natural-resource stock, the models with more than one type of physical capital of Hahn (1966) and Shell and Stiglitz (1967), and the model with capital and 
money of Shell et al. (1969) all exhibit saddlepoint phase diagrams. The same is true for the overlapping-generations models in Laitner $(1981,1984)$ with one asset but with households which live two or more periods.

In each paper above, the mixture of eigenvalues is related to the nature of the given model's initial conditions. Although history should determine stocks of physical assets at the start of an analysis, the same is not true for the initial relative prices of the stocks, which depend on present market conditions and on anticipated capital gains - variables which can 'jump' immediately after an unanticipated shock. If, therefore, a multi-asset model's stationary solution were a 'sink' point, the shortage of exogenous initial conditions would imply an indeterminacy problem - the model would not be able to tell us which of many convergent paths (consistent with the restricted set of fixed initial conditions) the economy would follow after a shock. On the other hand, with a saddlepoint phase diagram, if we limit our attention to stable paths - believing the actual economy does always return to a stationary state (at least after modest shocks) $)^{11}$ - there may be enough exogenous initial conditions to determine fully behavior within the diagram's stable arm. This typically is the case in the papers listed above - more stable eigenvalues would imply indeterminacy, but fewer would generally preclude convergence altogether. On the other hand, it is not difficult to find models having stationary solutions with the wrong constellation of eigenvalues for both determinacy and convergence - see, for instance, Calvo (1978) - so we must be careful to examine characteristic roots in individual cases [see condition (34) below]. ${ }^{12}$

In a one-good overlapping-generations model in which households have perfect foresight, history will fix each family's initial-period wealth. This wealth plus anticipated future wage and interest rates will determine the family's present saving. The aggregate present savings of all households governs the current evolution of the economy. The future wage and interest rates needed in an analysis of each family's initial-period savings behavior are strictly analogous to the starting relative stock prices in models with many physical assets, however. In other words, anticipations of future prices, or anticipations of the future capital-to-labor ratios which will generate them, can 'jump' after a current surprise. Again, ideally there will be enough stable eigenvalues for convergence from the exogenous initial conditions, but, to escape indeterminacy, not more.

\footnotetext{
"Ideally, we would be able to perform a global analysis showing that all unstable paths in a model's saddlepoint lead to eventual inconsistencies with our assumption of perfect foresight. With low-dimensional models this is sometimes possible - see, for instance, Shell and Stiglitz (1967). The present paper is limited to a local analysis.

${ }^{12}$ There are, of course, many 'optimal' growth models exhibiting saddlepoint phase diagrams for state and costate variables. Determinacy and convergence to a (possible) stationary state are important issues in such cases. However, we limit our discussion to decentralized models here.
} 
Returning to eq. (26), let $J$ be a diagonal matrix of the eigenvalues of $P$ arranged with roots of smallest modulus first. Let $E$ be a matrix of the corresponding eigenvectors, with

$$
E \cdot P=J \cdot E
$$

Define new vectors $y(i), i=1,2, \ldots$, with

$$
y(i) \equiv\left(\begin{array}{c}
y_{1}(i) \\
\vdots \\
y_{n-2}(i)
\end{array}\right) \equiv E \cdot\left(\begin{array}{c}
z_{i} \\
\vdots \\
z_{i+n-3}
\end{array}\right) .
$$

Define

$$
\tilde{q} \equiv E \cdot\left(\begin{array}{c}
0 \\
\vdots \\
0 \\
q
\end{array}\right)
$$

Then (26) implies [multiplying through by $E$ and using (27)]

$$
J \cdot\left(\begin{array}{c}
y_{1}(i) \\
\vdots \\
y_{n-2}(i)
\end{array}\right)+\tilde{q}=\left(\begin{array}{c}
y_{1}(i+1) \\
\vdots \\
y_{n-2}(i+1)
\end{array}\right) \text {. }
$$

Let

$$
J=\left(\begin{array}{cc}
J^{*} & 0 \\
0 & J^{* *}
\end{array}\right)
$$

with $J^{*}$ a submatrix of the eigenvalues in $J$ of modulus less than one. Define

$$
\tilde{q}=\left(\begin{array}{c}
\tilde{q}^{*} \\
\tilde{q}^{* *}
\end{array}\right), \quad y(i)=\left(\begin{array}{c}
y^{*}(i) \\
y^{* *}(i)
\end{array}\right), \quad E=\left(\begin{array}{c}
E^{*} \\
E^{* *}
\end{array}\right),
$$

where $q^{*}, y^{*}(i)$, and $E^{*}$ have the same number of rows as $J^{*}$. Let that number be $n_{s}$. Let the number of rows in $J^{* *}$ be $n_{e}$.

For our model to proceed along the stable arm of its saddlepoint, $y^{* *}(i)$ must follow its forward solution. In other words, we need

$$
y^{* *}(i)=-\left(J^{* *}\right)^{-1} \cdot\left[I-\left(J^{* *}\right)^{-1}\right]^{-1} \cdot \tilde{q}^{* *}, \text { all } i \geq 1,
$$

where $I$ is the $n_{e} \times n_{e}$ identity matrix. 
Returning to (24), the equations for rows 1 to $(n / 2)$ have so far been ignored. These equations define an $(n / 2) \times(n-1)$-dimensional matrix $\hat{Q}$ such that

$$
\hat{Q} \cdot\left(\begin{array}{c}
z_{0} \\
\vdots \\
z_{n-2}
\end{array}\right)=\left(\begin{array}{c}
H\left(t_{0}\right) \\
H\left(t_{1}\right) \\
\vdots \\
H\left(t_{(n / 2)-1}\right)
\end{array}\right) .
$$

This system follows from (22)-(23). It reflects information stemming from our knowledge of the behavior of the economy before time 0 - and locked in time- 0 family asset holdings; thus, it embodies the initial conditions given by history for our model..$^{13}$ Combining (31) and (32), if $\tilde{0}$ is a column vector of zeros, and if

then

$$
D \equiv\left(\begin{array}{ll}
\hat{Q} & \\
\tilde{0} & E^{* *}
\end{array}\right),
$$

If

$$
D \cdot\left(\begin{array}{c}
z_{0} \\
\vdots \\
z_{n-2}
\end{array}\right)=\left(\begin{array}{c}
H\left(t_{0}\right) \\
H\left(t_{1}\right) \\
\vdots \\
H\left(t_{(n / 2)-1}\right) \\
-\left(J^{* *}\right)^{-1} \cdot\left[I-\left(J^{* *}\right)^{-1}\right]^{-1} \cdot \tilde{q}^{* *}
\end{array}\right) .
$$

$$
(n / 2)+n_{e}=n-1,
$$

and assuming the matrix $D$ is non-singular, (33) uniquely determines $\left(z_{0}, \ldots, z_{n-2}\right)^{T}$. Subsequent $z_{i}$ values follow (uniquely) from (26). If $(n / 2)+$ $n_{e}<n-1$, there are too few eigenvalues of modulus greater than or equal to 1 for an assumption that the economy avoids explosive paths to determine a unique set of approximate multipliers. If $(n / 2)+n_{e}>n-1$, the stationary solution about which our linearizations are taken will not be stable (even with respect to our limited set of exogenous initial conditions); in such a case no bounded set of dynamic multipliers will exist in general. Fortunately, in each of our examples below (34) holds (and $D$ is invertible).

We can now fully characterize our practical solution $Z(t)$ for $\partial k_{t} / \partial \theta$, all $t \geq 0$. For $t$ with $t_{j} \leq t<t_{j+1}$, define

$$
\xi(t, j) \equiv\left(t-t_{j}\right) /\left(t_{j+1}-t_{j}\right)
$$

\footnotetext{
${ }^{13}$ Note: In the period $t<0$, anticipations of $w_{s}$ and $r_{s}$ for $s>0$ were, as it turns out, incorrect.
} 
Similarly, for $t_{j}<t<t_{j+1}$ define

$$
\begin{aligned}
& x_{i}^{*}(t, j) \equiv x_{i}\left(t_{j}\right), \quad \text { all } i=0, \ldots, n, \\
& x_{n+1}^{*}(t, j)=t_{j}+T+h, \\
& A_{i}^{*}=A_{i}, \quad \text { all } i=0, \ldots, n-1, \\
& A_{n}^{*}=h / 3+h / 2, \\
& A_{n+1}^{*}=h / 2, \\
& x_{0}^{* *}(t, j)=t_{j+1}-T-h, \\
& x_{i}^{* *}(t, j)=x_{i-1}\left(t_{j+1}\right), \quad \text { all } i=1, \ldots, n+1, \\
& A_{0}^{* *}=h / 2, \\
& A_{1}^{* *}=h / 3+h / 2, \\
& A_{i}^{* *}=A_{i-1}, \quad \text { all } i=2, \ldots, n+1 .
\end{aligned}
$$

Then given $z_{i}, i \geq 0$, as generated above, set

$$
\begin{aligned}
& Z(t) \equiv {[1-\xi(t, j)] \cdot \sum_{i=0}^{n+1} G\left(t, x_{i}^{*}(t, j)\right) \cdot A_{i}^{*} \cdot z_{x_{i}^{*}(t, j)} } \\
&+\xi(t, j) \cdot \sum_{i=0}^{n+1} G\left(t, x_{i}^{* *}(t, j)\right) \cdot A_{i}^{* *} \cdot z_{x_{i}^{* *}(t, j)}+H(t), \\
& \quad \text { all } t_{j} \leq t<t_{j+1} .
\end{aligned}
$$

Note that

$$
z_{i}=Z\left(t_{i}\right), \text { all } j \geq 0,
$$

[recall the zero conditions for $G(\cdot)$ ] and that $Z(\cdot)$ is defined in such a way that it will be a continuous function of $t$ (the latter point becoming important in section 5).

\section{Errors of approximation}

Let $\zeta(t)$, all $t \geq 0$, be a solution to (18), and let $Z(\cdot)$ be as in (35). Then this section introduces a procedure for bounding the errors of approximation 
$|Z(t)-\zeta(t)|$, all $t$. We will assume $G(\cdot)$ and $H(\cdot)$ are four times continuously differentiable. ${ }^{14}$

Given any $\zeta:[0, \infty) \rightarrow R^{1}$ for which the left-hand side of (21) exists, let $\sigma(\cdot)$ be the error in (21) of replacing an integral with a summation:

$$
\begin{aligned}
\sigma & (t, \zeta(\cdot)) \\
\equiv & \int_{0}^{\infty} G(t, x) \cdot \zeta(x) \mathrm{d} x-[1-\xi(t, j)] \\
& \cdot \sum_{i=0}^{n+1} G\left(t, x_{i}^{*}(t, j)\right) \cdot A_{i}^{*} \cdot \zeta\left(x_{i}^{*}(t, j)\right) \\
& -\xi(t, j) \cdot \sum_{i=0}^{n+1} G\left(t, x_{i}^{* *}(t, j)\right) \cdot A_{i}^{* *} \cdot \zeta\left(x_{i}^{* *}(t, j)\right) \\
= & {[1-\xi(t, j)] } \\
& \cdot\left\{\int_{0}^{\infty} G(t, x) \cdot \zeta(x) \mathrm{d} x-\sum_{i=0}^{n+1} G\left(t, x_{i}^{*}(t, j)\right) \cdot A_{i}^{*} \cdot \zeta\left(x_{i}^{*}(t, j)\right)\right\} \\
& +\xi(t, j) \cdot\left\{\int_{0}^{\infty} G(t, x) \cdot \zeta(x) \mathrm{d} x\right. \\
& \left.-\sum_{i=0}^{n+1} G\left(t, x_{i}^{* *}(t, j)\right) \cdot A_{i}^{* *} \cdot \zeta\left(x_{i}^{* *}(t, j)\right)\right\} .
\end{aligned}
$$

Davis and Rabinowitz (1975, p. 46) provide an upper limit for $\sigma(\cdot):{ }^{15}$ if $b^{*}(i, t, \zeta(\cdot))$ bounds the fourth derivative of the integrand on the left side of (21) for $x \in\left[x_{i-2}(t), x_{i}(t)\right]$

$$
b(i, t, \zeta(\cdot)) \equiv h^{5} \cdot b^{*}(i, t, \zeta(\cdot)) / 180
$$

\footnotetext{
${ }^{14}$ If this were not true, provided $G(\cdot)$ and $H(\cdot)$ are at least continuous, we can approximate them with $C^{4}$ functions to any degree of accuracy on the compact set $\Omega$ defined in section 5 - see, for instance, Lorentz (1966, theorem 6, p. 10). We could then perform our analysis on the approximations, with errors proportional to the approximation accuracies - see the analysis below.

${ }^{15}$ Our $\sigma(\cdot)$ is really a weighted average of two approximations, but the weights sum to 1 . As indicated in note 10 , for simplicity we omit the (single interval) trapezoidal-approximation error in each case with $t \neq t_{j}$.
} 
bounds the error of Simpson's approximation for the same interval. Hence,

$$
|\sigma(t, \zeta(\cdot))| \leq B(t, \zeta(\cdot)) \equiv \sum_{i=1}^{(n / 2)+1} h^{5} \cdot b^{*}(2 i, t, \zeta(\cdot)) / 180
$$

Differentiating both sides of (18) $i$ times with respect to $t$,

$$
\zeta^{(i)}(t)=\int_{0}^{\infty}\left[\partial^{i} G(t, x) / \partial t^{i}\right] \cdot \zeta(x) \mathrm{d} x+H^{(i)}(t),
$$

[where $\zeta^{(i)}(t) \equiv \partial^{i} \zeta(t) / \partial t^{i}$ and $H^{(i)}(t) \equiv \partial^{i} H(t) / \partial t^{i}$ ]. Thus, $\zeta(\cdot)$ is four times continuously differentiable [if $G(\cdot)$ and $H(\cdot)$ are]. We have

$$
\begin{aligned}
b^{*}(i, t, \zeta(\cdot)) \equiv & \max _{x_{i-2}(t) \leq x \leq x_{i}(t)} \mid\left[\partial^{4} G(t, x) / \partial x^{4}\right] \cdot \zeta(x) \\
& +4 \cdot\left[\partial^{3} G(t, x) / \partial x^{3}\right] \cdot \zeta^{(1)}(x) \\
& +6 \cdot\left[\partial^{2} G(t, x) / \partial x^{2}\right] \cdot \zeta^{(2)}(x) \\
& +4 \cdot[\partial G(t, x) / \partial x] \cdot \zeta^{(3)}(x) \\
& +G(t, x) \cdot \zeta^{(4)}(x) \mid \\
\leq & \max _{x_{i-2}(t) \leq x \leq x_{i}(t)}\left\{\left|\partial^{4} G(t, x) / \partial x^{4}\right| \cdot|\zeta(x)|\right. \\
& \left.+\cdots+|G(t, x)| \cdot\left|\zeta^{(4)}(x)\right|\right\} .
\end{aligned}
$$

As explained in section 3, we are interested in non-explosive adjustment paths. That is, we care about sequences of multipliers which are bounded, say, by $\zeta^{*}<\infty$ :

$$
\sup _{t \geq 0}|\zeta(t)|=\zeta^{*}<\infty
$$

Eq. (37) then yields

$$
\left|\zeta^{(i)}(t)\right| \leq \zeta^{*} \cdot \int_{0}^{\infty}\left|\partial^{i} G(t, x) / \partial t^{i}\right| \cdot \mathrm{d} x+\left|H^{(i)}(t)\right| .
$$

Thus, (38) implies there exist non-negative functions $\tau_{0}(t)$ and $\tau_{1}(t)$, dependent only on the properties of $G(\cdot)$ and $H(\cdot)$, with, for all $\zeta(\cdot)$ satisfying (39),

$$
|\sigma(t, \zeta(\cdot))| \leq B(t, \zeta(\cdot)) \leq \tau_{0}(t) \cdot \zeta^{*}+\tau_{1}(t), \quad \text { all } t \geq 0
$$

Define

$$
\tau_{0}^{*} \equiv \tau_{0}\left(t_{n / 2}\right) \text { and } \tau_{1}^{*} \equiv \tau_{1}\left(t_{n / 2}\right) .
$$


Property (19) and eq. (17) imply

$$
\tau_{0}\left(t_{i}\right)=\tau_{0}^{*} \text { and } \tau_{1}\left(t_{i}\right)=\tau_{1}^{*}, \quad \text { all } i \geq n / 2
$$

Define

$$
\Delta\left(t_{j}, \zeta(\cdot)\right) \equiv \zeta\left(t_{j}\right)-z_{j}
$$

Subtracting each side of (22) from each side of (18), we have

$$
\begin{aligned}
\Delta\left(t_{j}, \zeta(\cdot)\right)= & \sigma\left(t_{j}, \zeta(\cdot)\right)+\sum_{i=1}^{n-1} G\left(t_{j}, x_{i}\left(t_{j}\right)\right) \\
& \cdot A_{i} \cdot \Delta\left(x_{i}\left(t_{j}\right), \zeta(\cdot)\right), \quad \text { all } j \geq 0 .
\end{aligned}
$$

Since $\zeta(0)=z_{0}=0, \Delta(0, \zeta(\cdot))=0$. Eq. (43) has almost exactly the same form as (22) and can be solved in virtually the same way.

Provided (34) holds and $D$ is invertible [see (33)], section 3 shows how to generate a unique bounded sequence $z_{i}$. If (39) holds for some $\zeta^{*}<\infty$ and some solution $\zeta(t)$ for $(18), \sigma(t, \zeta(\cdot))$ is bounded. Condition (34) and $D$ non-singular then yield a unique solution, via (43), for $\Delta\left(t_{j}, \zeta(\cdot)\right)$, all $j \geq 0$ [in terms of $\sigma(t, \zeta(\cdot))$ ]. Letting $E^{*}, E^{* *}, J^{*}, J^{* *}$, and $K$ be as in section 3 , if

$$
\begin{aligned}
\pi \equiv-\left(J^{* *}\right)^{-1} \cdot E^{* *} \cdot\left(\begin{array}{c}
0 \\
\vdots \\
0 \\
\sigma\left(t_{n / 2}, \zeta(\cdot)\right)
\end{array}\right) \\
-\left(J^{* *}\right)^{-2} \cdot E^{* *}\left(\begin{array}{c}
0 \\
\vdots \\
\sigma\left(t_{(n / 2)+1}, \zeta(\cdot)\right)
\end{array}\right)+\cdots,
\end{aligned}
$$

then

$$
D \cdot\left(\begin{array}{c}
\Delta(0, \zeta(\cdot)) \\
\vdots \\
\Delta\left(t_{n-2}, \zeta(\cdot)\right)
\end{array}\right)=\left(\begin{array}{c}
0 \\
\sigma\left(t_{1}, \zeta(\cdot)\right) \\
\vdots \\
\sigma\left(t_{(n / 2)-1}, \zeta(\cdot)\right) \\
\pi
\end{array}\right) .
$$

So, if

$$
\Pi \equiv\left|J^{* *-1}\right| \cdot\left|\left[I-\left|J^{* *-1}\right|\right]^{-1}\right| \cdot\left|E^{* *}\right| \cdot\left[\begin{array}{c}
0 \\
\vdots \\
0 \\
1
\end{array}\right] \cdot\left(\tau_{0}^{*} \cdot \zeta^{*}+\tau_{1}^{*}\right)
$$


where for $M=\left[m_{i j}\right],|M| \equiv\left[\left|m_{i j}\right|\right]$,

$$
\begin{aligned}
\left(\begin{array}{c}
|\Delta(0, \zeta(\cdot))| \\
\vdots \\
\left|\Delta\left(t_{n-2}, \zeta(\cdot)\right)\right|
\end{array}\right) & \leq\left|D^{-1} \cdot\left(\begin{array}{c}
0 \\
\sigma\left(t_{1}, \zeta(\cdot)\right) \\
\vdots \\
\pi
\end{array}\right)\right| \\
& \leq\left|D^{-1}\right| \cdot\left(\begin{array}{c}
\tau_{0}\left(t_{1}\right) \cdot \zeta^{*}+\tau_{1}\left(t_{1}\right) \\
\vdots \\
\tau_{0}\left(t_{(n / 2)-1}\right) \cdot \zeta^{*}+\tau_{1}\left(t_{(n / 2)-1}\right) \\
\Pi
\end{array}\right) .
\end{aligned}
$$

Let the vector on the right-hand side of $\left|D^{-1}\right|$ be

$$
\Pi^{*} \cdot \zeta^{*}+\Pi^{* *}
$$

(where each element of $\Pi^{*}$ and $\Pi^{* *}$ is non-negative). Then, since $\zeta\left(t_{i}\right)=$ $\Delta_{i}+z_{i}$,

$$
\left(\begin{array}{c}
\left|\zeta\left(t_{0}\right)\right| \\
\vdots \\
\left|\zeta\left(t_{n-2}\right)\right|
\end{array}\right) \leq\left|D^{-1}\right| \cdot \Pi^{*} \cdot \zeta^{*}+\left|D^{-1}\right| \cdot \Pi^{* *}+\left(\begin{array}{c}
\left|z_{0}\right| \\
\vdots \\
\left|z_{n-2}\right|
\end{array}\right)
$$

We can use (45) and $|P|$ to bound $\left|\Delta\left(t_{i}, \zeta(\cdot)\right)\right|$, all $i \geq n-1$, and, hence, $\left|\zeta\left(t_{i}\right)\right|$, all $i \geq n-1$.

For $t$ with $t_{j}<t<t_{j+1}$

$$
\begin{aligned}
|\zeta(t)| \leq & |Z(t)|+|\zeta(t)-Z(t)| \leq|Z(t)|+|\sigma(t, \zeta(\cdot))| \\
& +[1-\xi(t, j)] \cdot\left|\sum_{i=0}^{n+1} G\left(t, x_{i}^{*}(t, j)\right) \cdot A_{i}^{*} \cdot \Delta\left(x_{i}^{*}(t, j), \zeta(\cdot)\right)\right| \\
& +\xi(t, j) \cdot\left|\sum_{i=0}^{n+1} G\left(t, x_{i}^{* *}(t, j)\right) \cdot A_{i}^{* *} \cdot \Delta\left(x_{i}^{* *}(t, j), \zeta(\cdot)\right)\right| .
\end{aligned}
$$

On the right-hand side of (47), $Z(t)$ is known [see (35)], $|\sigma(\cdot)|$ is bounded in (41), and each $\Delta(\cdot)$ is bounded in the preceding paragraph. 
Ultimately, each inequality for $|\zeta(t)|$ has the form

$$
|\zeta(t)| \leq c(t) \cdot \zeta^{*}+d(t)
$$

with $c(\cdot)$ and $d(\cdot) \geq 0$ and independent of $\zeta(\cdot)$. Suppose, for some $\varepsilon>0$,

$$
c(t) \leq 1-\varepsilon, \text { all } t \geq 0 \text {. }
$$

Define

$$
\zeta^{* *} \equiv \sup _{t \geq 0}|d(t) /[1-c(t)]|
$$

Notice that we can hope to communicate $\zeta^{* *}$, which is independent of $\zeta^{*}$. Fortunately, $\zeta^{* *}$ is an upper bound for $\zeta^{*}$.

Proposition 1. Suppose (18) has a solution $\zeta(t)$ which is bounded as in (39). Suppose for some grid and some $\varepsilon>0$, (34) holds, $D$ is invertible, and (49) holds. Then $\zeta^{* *}$ from $(50)$ bounds $\zeta^{*}$ in (39).

Proof. Let (49) hold for some $\varepsilon>0$. Define

$$
\alpha(t) \equiv d(t) /[1-c(t)]
$$

From (48) and (39),

So

$$
\sup _{t \geq 0}\left\{c(t) \cdot \zeta^{*}+d(t)\right\} \geq \zeta^{*}
$$

$$
\begin{aligned}
0 & \geq \sup _{t \geq 0}\left\{\zeta^{*}-c(t) \cdot \zeta^{*}-d(t)\right\} \\
& =\sup _{t \geq 0}\left\{[1-c(t)] \cdot\left[\zeta^{*}-\alpha(t)\right]\right\} \\
& \geq \varepsilon \cdot \sup _{t \geq 0}\left\{\zeta^{*}-\alpha(t)\right\},
\end{aligned}
$$

or

$$
\sup _{t \geq 0}\left\{\zeta^{*}-\alpha(t)\right\} \leq 0
$$

In the former case, because $\varepsilon>0$,

$$
\sup _{t \geq 0}\left\{\zeta^{*}-\alpha(t)\right\}=0 .
$$

Thus,

$$
\zeta^{* *}=\sup _{t \geq 0} \alpha(t) \geq \zeta^{*}
$$


In summary, if the first suppositions of Proposition 1 are valid, we can solve for $z_{i}$ and $Z(t)$. Employing the matrices used to generate $z_{i}$, a finite number of steps may verify (49) and yield $\zeta^{* *}$. Provided $\zeta^{* *}<\infty$, using $\zeta^{* *}$ in place of $\zeta^{*}$ in (45), we can bound the approximation errors $\left|\zeta\left(t_{i}\right)-z_{i}\right|$, all $i=0, \ldots$, $n-2$. As indicated, the analysis can easily be extended beyond $t_{n-2}$. Using the terms other than $|Z(t)|$ on the right side of (47), we can also bound $|\zeta(t)-Z(t)|$.

\section{Exact solutions}

Sections 3-4 study approximations - assuming that a bounded solution $\zeta(\cdot)$ for (17)-(18) exists. This section shows that the existence and uniqueness of $\zeta(\cdot)$ follow from the suppositions employed in section 4 if $\zeta^{* *}<\infty$ in (50).

Condition (19) indicates that we need only worry about the properties of $G(\cdot)$ and $H(\cdot)$ on the set

$$
\Omega \equiv\{(t, x): 0 \leq t \leq T ; 0 \leq x \leq t+T\}
$$

Our result is:

Proposition 2. Let $G(\cdot)$ and $H(\cdot) \in C^{4}$ and obey (19)-(20). Suppose $\zeta^{* *}<\infty$ in (50), (34) holds, $D$ is invertible, and (49) holds for some $\varepsilon>0$. Then (18) has a bounded solution.

Proof. Let the suppositions above hold. Let $z_{i}, i \geq 0$, and $Z(t), t \geq 0$, be as in section 3.

Step 1. Replace $\sigma\left(t_{j}, \zeta(\cdot)\right)$ in (43) with $\sigma\left(t_{j}, \zeta(\cdot)\right)+H\left(t_{j}\right)$. Replace $|Z(t)|$ on the right-hand side of (47) with $|H(t)|$. Then we must replace $d(t)$ in (48) with, say, $\bar{d}(t)$. Define

$$
\bar{\zeta} \equiv \sup _{t \geq 0}|\bar{d}(t) /[1-c(t)]|
$$

Then (49) and $\zeta^{* *}<\infty$ imply $\bar{\zeta}<\infty$.

Step 2. Define a set

$$
\mathscr{F} \equiv\{\zeta:[0, \infty) \rightarrow[-\bar{\zeta}, \bar{\zeta}] \mid, \zeta(\cdot) \text { continuous }\}
$$

For each $\zeta(\cdot) \in \mathscr{F}, \sigma(t, \zeta(\cdot))$, all $t$, is well defined. For any $\zeta(\cdot) \in \mathscr{F}$, let 
$z(0, \zeta(\cdot))=0$ and let $z\left(t_{j}, \zeta(\cdot)\right), j \geq 1$, satisfy

$$
\begin{aligned}
z\left(t_{j}, \zeta(\cdot)\right)= & \sigma\left(t_{j}, \zeta(\cdot)\right)+H\left(t_{j}\right) \\
& +\sum_{i=1}^{n-1} G\left(t_{j}, x_{i}\left(t_{j}\right)\right) \cdot A_{i} \cdot z\left(x_{i}\left(t_{j}\right), \zeta(\cdot)\right) .
\end{aligned}
$$

Comparing (51) with (43), the analysis following the latter shows (51) has a unique solution. For $t_{j} \leq t<t_{j+1}$, let

$$
\begin{aligned}
Z(t, \zeta(\cdot)) \equiv & {[1-\xi(t, j)] \cdot \sum_{i=0}^{n+1} G\left(t, x_{i}^{*}(t, j)\right) \cdot A_{i}^{*} \cdot z\left(x_{i}^{*}(t, j), \zeta(\cdot)\right) } \\
& +\xi(t, j) \cdot \sum_{i=0}^{n+1} G\left(t, x_{i}^{* *}(t, j)\right) \cdot A_{i}^{* *} \\
& \cdot z\left(x_{i}^{* *}(t, j), \zeta(\cdot)\right)+\sigma(t, \zeta(\cdot))+H(t) .
\end{aligned}
$$

The analysis following (43) shows

$$
|Z(t, \zeta(\cdot))| \leq \bar{\zeta}, \quad \text { all } t \geq 0
$$

Step 3. Fix any $t \geq 0$, any $\zeta(\cdot) \in \mathscr{F}$, and any $\delta>0 . G(\cdot)$ and $H(\cdot)$ are continuous on $\Omega$, hence uniformly continuous and bounded above by, say, $\bar{G}$ and $\bar{H}$. So, there exists $\phi(\delta)>0$, such that $t, t^{\prime} \geq 0$ and $\left|t^{\prime}-t\right|<\phi(\delta)$ implies $\left|G\left(t^{\prime}, x\right)-G(t, x)\right|<\delta$ and $\left|H\left(t^{\prime}\right)-H(t)\right|<\delta$, all $x \in[t-T, t+T]$. Thus $\left|t^{\prime}-t\right|<\phi(\delta)$ implies

$$
\begin{aligned}
\left|\sigma\left(t^{\prime}, \zeta(\cdot)\right)-\sigma(t, \zeta(\cdot))\right| \leq & 2 T \delta \bar{\zeta}+(n+2) \delta 4 h \bar{\zeta} / 3 \\
& +(n+2) \phi(\delta) \cdot \bar{G} \cdot 4 h \bar{\zeta} /(3 h),
\end{aligned}
$$

[note that the last term follows from the definition of $\xi(\cdot)$ ]. Similarly, since (52) shows $\left|z\left(t_{j}, \zeta(\cdot)\right)\right| \leq \bar{\zeta}$,

$$
\begin{aligned}
\left|Z\left(t^{\prime}, \zeta(\cdot)\right)-Z(t, \zeta(\cdot))\right| \leq & \phi(\delta) \cdot(n+2) \cdot \bar{G} \cdot 4 h \bar{\zeta} /(3 h) \\
& +(n+2) \cdot \delta 4 h \bar{\zeta} / 3 \\
& +\left|\sigma\left(t^{\prime}, \zeta(\cdot)\right)-\sigma(t, \zeta(\cdot))\right|+\delta .
\end{aligned}
$$


Thus, for any $\zeta(\cdot) \in \mathscr{F}, Z(t, \zeta(\cdot))$ is a continuous function of $t$. In fact, for any $\eta>0$ we can find some $\phi^{*}(\eta)>0$ with $\left|t^{\prime}-t\right|<\phi^{*}(\eta)$ and $t, t^{\prime} \geq 0$ implying

$$
\left|Z\left(t^{\prime}, \zeta(\cdot)\right)-Z(t, \zeta(\cdot))\right|<\eta, \quad \text { all } \zeta(\cdot) \in \mathscr{F} .
$$

Let

$$
\begin{array}{r}
\mathscr{F}^{*} \equiv\left\{\zeta(\cdot) \in \mathscr{F}: t, t^{\prime} \geq 0 \text { and }\left|t^{\prime}-t\right|<\phi^{*}(\eta)\right. \\
\text { imply } \left.\left|\zeta\left(t^{\prime}\right)-\zeta(t)\right|<\eta\right\} .
\end{array}
$$

Define

$$
\Phi(\zeta(\cdot))=\zeta^{*}(\cdot) \text { with } \zeta^{*}(t) \equiv Z(t, \zeta(\cdot)), \text { all } t \geq 0 .
$$

Then we have shown

$$
\Phi(\mathscr{F}) \subset \mathscr{F}^{*}
$$

$\mathscr{F}^{*}$ is clearly convex and equicontinuous [see Munkres (1975, p. 276)]. Hence, its closure is compact in the topology induced by the uniform norm [see Munkres $\left(1975\right.$, p. 290)]. In fact, we can see that closure $\left(\mathscr{F}^{*}\right)=\mathscr{F}^{*}$ in our case.

Step 4. Define

$$
\left\|\zeta(\cdot)-\zeta^{*}(\cdot)\right\|=\sup _{t \geq 0}\left|\zeta(t)-\zeta^{*}(t)\right|
$$

Then

$$
\begin{aligned}
\left|\sigma(t, \zeta(\cdot))-\sigma\left(t, \zeta^{*}(\cdot)\right)\right| & \leq\{2 T \bar{G}+(n+2) \bar{G} 4 h / 3\} \cdot\left\|\zeta(\cdot)-\zeta^{*}(\cdot)\right\| \\
& \equiv c_{0} \cdot\left\|\zeta(\cdot)-\zeta^{*}(\cdot)\right\|, \quad \text { all } t \geq 0 .
\end{aligned}
$$

The analysis of (43)-(48) shows

$$
\left|Z(t, \zeta(\cdot))-Z\left(t, \zeta^{*}(\cdot)\right)\right| \leq c(t) \cdot c_{0} \cdot\left\|\zeta(\cdot)-\zeta^{*}(\cdot)\right\|, \quad \text { all } t \geq 0 .
$$

Thus, $\Phi(\cdot)$ is continuous on $\mathscr{F}$.

Step 5. The preceding steps and Schauder's fixed point theorem [see Berge (1963, p. 252)] guarantee the existence of $\zeta(\cdot) \in \mathscr{F}^{*} \subset \mathscr{F}$ with $\zeta(\cdot)=$ $\Phi(\zeta(\cdot))$ - in other words, of a bounded solution to (18). 
Table 2

Dynamic multipliers (multipliers w.r.t. $\theta$ for $k$ and errors of approximation).

\begin{tabular}{|c|c|c|c|c|c|c|c|c|}
\hline \multirow[b]{2}{*}{ Time } & \multicolumn{2}{|c|}{$\begin{array}{c}\text { Example 1 } \\
(\rho=1.0, \beta=-1.0, \\
\left.\tau_{w}=0.30, \tau_{r}=0.30\right)^{\mathrm{a}} \\
\end{array}$} & \multicolumn{2}{|c|}{$\begin{array}{c}\text { Example 2 } \\
(\rho=1.0, \beta=0.0 \\
\left.\tau_{w}=0.3, \tau_{r}=0.3\right)\end{array}$} & \multicolumn{2}{|c|}{$\begin{array}{c}\text { Example 3 } \\
(\rho=1.0, \beta=-1.0, \\
\left.\tau_{w}=0.28, \tau_{r}=0.40\right)\end{array}$} & \multicolumn{2}{|c|}{$\begin{array}{c}\text { Example 4 } \\
(\rho=1.0, \beta=0.0 \\
\left.\tau_{w}=0.28, \tau_{w}=0.40\right)\end{array}$} \\
\hline & Mult. & Error & Mult. & Error & Mult. & Error & Mult. & Error \\
\hline 0 & 0.0000 & 0.0000 & 0.0000 & 0.0000 & 0.0000 & 0.0000 & 0.0000 & 0.0000 \\
\hline 1 & 0.1960 & 0.0019 & 0.4898 & 0.0022 & 0.1768 & 0.0019 & 0.4123 & 0.0020 \\
\hline 2 & 0.3605 & 0.0123 & 0.9219 & 0.0145 & 0.3237 & 0.0121 & 0.7742 & 0.0128 \\
\hline 3 & 0.5200 & 0.0154 & 1.3307 & 0.0180 & 0.4661 & 0.0152 & 1.1158 & 0.0160 \\
\hline 4 & 0.6456 & 0.0146 & 1.6797 & 0.0181 & 0.5768 & 0.0144 & 1.4056 & 0.0160 \\
\hline 5 & 0.7607 & 0.0244 & 2.0003 & 0.0295 & 0.6778 & 0.0238 & 1.6708 & 0.0260 \\
\hline 6 & 0.8389 & 0.0232 & 2.2590 & 0.0305 & 0.7443 & 0.0225 & 1.8824 & 0.0269 \\
\hline 7 & 0.9443 & 0.0334 & 2.5421 & 0.0407 & 0.8369 & 0.0326 & 2.1157 & 0.0360 \\
\hline 8 & 1.0122 & 0.0284 & 2.7624 & 0.0354 & 0.8946 & 0.0278 & 2.2947 & 0.0312 \\
\hline 9 & 1.0804 & 0.0452 & 2.9720 & 0.0538 & 0.9528 & 0.0440 & 2.4649 & 0.0474 \\
\hline 10 & 1.1087 & 0.0460 & 3.1170 & 0.0623 & 0.9742 & 0.0440 & 2.5796 & 0.0544 \\
\hline 11 & 1.1825 & 0.0575 & 3.3172 & 0.0708 & 1.0382 & 0.0556 & 2.7423 & 0.0621 \\
\hline 12 & 1.2169 & 0.0446 & 3.4526 & 0.0558 & 1.0659 & & 494 & 0.0489 \\
\hline 13 & 1.2575 & 0.0667 & 3.5888 & 0.0806 & 1.0997 & 0.0643 & 2.9576 & 0.0705 \\
\hline 14 & 1.2568 & 0.0623 & 3.6587 & 0.0853 & 1.0954 & 0.0592 & 3.0093 & 0.0740 \\
\hline 15 & 1.3116 & 0.0747 & 3.8033 & 0.0932 & 1.1427 & 0.0717 & 3.1254 & 0.0812 \\
\hline 16 & 1.3260 & 0.0567 & 3.8820 & 0.0716 & 1.1530 & 0.0545 & 3.1853 & 0.0624 \\
\hline 17 & 1.3500 & 0.0814 & 3.9688 & 0.1004 & 1.1724 & 0.0781 & 3.2525 & 0.0873 \\
\hline 18 & 1.3320 & 0.0737 & 3.9887 & 0.1020 & 1.1533 & 0.0696 & 3.2629 & 0.0880 \\
\hline 19 & 1.3748 & 0.0862 & 4.0953 & 0.1093 & 1.1903 & 0.0823 & 3.3476 & 0.0947 \\
\hline 20 & 1.3770 & 0.0652 & 4.1357 & 0.0832 & 1.1901 & 0.0623 & 3.3761 & 0.0721 \\
\hline 50 & 1.2597 & 0.0865 & 4.1749 & 0.1276 & 1.0686 & 0.0802 & 3.3497 & 0.1075 \\
\hline$\infty$ & 1.285 & & 4.202 & & 1.091 & & 3.374 & \\
\hline
\end{tabular}

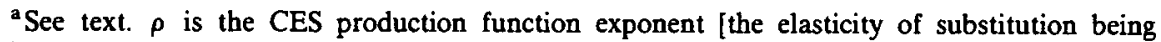
$1 /(1+\rho)]$.

Uniqueness follows more easily.

Proposition 3. If the suppositions of Proposition 1 hold and if in $(50) \zeta^{* *}<\infty$, any bounded solution $\zeta(\cdot)$ of $(18)$ is unique (among bounded solutions).

Proof. Let $\zeta_{1}(\cdot)$ and $\zeta_{2}(\cdot)$ be bounded solutions for (18). Then for any $\lambda$, $\zeta_{3}(\cdot) \equiv \lambda \cdot \zeta_{1}(\cdot)+(1-\lambda) \cdot \zeta_{2}(\cdot)$ satisfies (18) and is bounded. However, if $\zeta_{1}(t) \neq \zeta_{2}(t)$, any $t \geq 0$, we can choose $\lambda$ with $\left|\zeta_{3}(t)\right|>\zeta^{* *}$ - a violation of Proposition 1. Hence, $\zeta_{1}(t)=\zeta_{2}(t)$, all $t \geq 0$.

\section{Examples}

Section 2 outlines a specific model. We now subject that model to the procedures discussed in sections 3-5. Table 2 presents the resulting transition paths. In all cases we choose a numerical grid width of two years (see, however, section 7). 
Our procedure is to evaluate $G(\cdot)$ and $H(\cdot)$ at the grid points in the set $\Omega$; to use the evaluations to generate matrices $P$ and $\hat{Q}$; to find the eigenvalues and eigenvectors of $P$; to check (34) and the non-singularity of $D$; to calculate $z_{i}$, all $i \geq 0$; to calculate $Z(t)$, all $t=0,1,2, \ldots$; to derive $\tau_{0}(\cdot)$ and $\tau_{1}(\cdot)$ for $t=0,1, \ldots$ using numerical derivatives; to check (49) and $\zeta^{* *}<\infty$; and to calculate bounds for $|\zeta(t)-Z(t)|$, all $t=0,1, \ldots$. We then use

$$
|\zeta(t)| \leq|\zeta(t)-Z(t)|+|Z(t)|
$$

to develop year-by-year upper bounds for $\zeta(\cdot)$. These, in turn, yield sharper values for $\tau_{0}(\cdot)$ and $\tau_{1}(\cdot)$ - see (37), (38), and (40). On the basis of the latter, we can recalculate bounds for $|\zeta(t)-Z(t)|$ and then $\zeta(\cdot)$. Table 2 displays figures obtained after five iterations.

The table presents four sample calculations, using yearly figures from formula (35). For the type of shock we consider, a permanent change in the constant multiplying the aggregate production function, half-lives for convergence to the new stationary state fall in the range of 3-6 years. In every example, all of our checks [i.e., eq. (34), the invertibility of $D,(49)$ for some $\varepsilon>0$, and $\left.\zeta^{* *}<\infty\right]$ were satisfied.

Since the maximum errors of approximation are all under 10 percent (and in early years under 5 percent), a finer grid does not seem to be required. Notice that our grid points fall at even numbered years and that multipliers evaluated there tend to be the most accurate.

Computation times on an Amdahl 5860 computer averaged about 20 seconds per example.

\section{Conclusions}

We have presented a way of analyzing the dynamic behavior of a continuous-time overlapping-generations model. The examples in section 6 illustrate that the procedure can be successfully implemented in practice and that its computer-time demands are fairly modest.

Because the errors of approximation derived from our examples are related to a relatively high power of the chosen grid width [see (36)], although the tables in section 6 indicate two-year intervals perform quite well, further widening would seem inadvisable if we continue to employ Simpson's rule. Most more sophisticated quadrature rules will be less satisfactory on our coarse grid. One exception, however, is the trapezoidal rule with endpoint corrections - see David and Rabinowitz (1975, p. 105). This rule would be more elaborate to program and would require more attention to the differentiability (with respect to $t$ ) of $G(\cdot)$. However, if it were employed, it seems likely that we could increase our grid width to 3-4 years. In special applications involving elaborations of our economic model, the corresponding reduction in the dimensions of the matrices $D$ and $P$ might be worthwhile. 


\section{References}

Aoki, M., 1980, Note on comparative dynamic analysis, Econometrica 48, 1319-1325.

Arthur, W.B. and G. McNicoll, 1978, Samuelson, population and intergenerational transfers, International Economic Review 19, 241-246.

Auerbach, A.J. and L.J. Kotlikoff, Social security and the economics of the demographic transition, in: H.J. Aaron and G. Burtless, eds., Retirement and economic behavior (Brookings Institution, Washington, DC) 255-278.

Berge, C. (transl. E.M. Patterson), 1963, Topological spaces (Oliver \& Boyd, London).

Calvo, G., 1978, On the indeterminacy of interest rates and wages with perfect foresight, Journal of Economic Theory 19, 321-337.

Coppel, W.A., 1965, Stability and asymptotic behavior of differential equations (D.C. Heath \& Co., Boston, MA).

David, P.J. and P. Rabinowitz, 1975, Methods of numerical integration (Academic Press, New York).

Hahn, F.H., 1966, Equilibrium dynamics with heterogeneous capital goods, Quarterly Journal of Economics 80, 633-646.

Kantorovich, L.V. and V.I. Krylov (transl. C.D. Benster), 1958, Approximate methods of higher analysis (Noordhoff, Groningen, Netherlands).

Katzner, D., 1970, Static demand theory (Macmillan, New York).

Kotlikoff, L.J., 1979, Social security and equilibrium capital intensity, Quarterly Journal of Economics 93, 233-254.

Laitner, J.P., 1981, The stability of steady states in perfect foresight models, Econometrica 49, 319-333.

Laitner, J.P., 1982, Monopoly and long-run capital accumulation, Bell Journal of Economics 13, $143-157$.

Laitner, J.P., 1984, Transition time paths for overlapping-generations models, Journal of Economic Dynamics and Control 7, 111-129.

Lorentz, G.G., 1966, Approximation of functions (Holt, Rinehart, and Winston, New York).

Lucas, R.E., 1969, Labor-capital substitution in U.S. manufacturing, in: A.C. Harberger and M.J. Baily, eds., The taxation of income from capital (Brookings Institution, Washington, DC) 223-274.

Munkres, J.R., 1975, Topology (Prentice-Hall, Englewood Cliffs, NJ).

Musgrave, J., 1982, Fixed reproducible tangible wealth in the United States, Survey of Current Business 62, 33-36.

Shell, K. and J.E. Stiglitz, 1967, The allocation of investment in a dynamic economy, Quarterly Journal of Economics 81, 592-609.

Shell, K., M. Sidrauski and J.E. Stiglitz, Capital gains, income and savings, Review of Economic Studies 36, 15-26.

Solow, R.M., 1956, A contribution to the theory of economic growth, Quarterly Journal of Economics 81, 65-94.

Stiglitz, J.E., 1974, Growth with exhaustible resources: The competitive economy, Review of Economic Studies symposium issue, 139-152.

Summer, L.H., 1981, Capital taxation and accumulation in a life cycle growth model, American Economic Review 71, 533-544.

Tobin, J., 1967, Life cycle saving and balanced growth, in: W. Fellner, ed., Ten economic essays in the tradition of Irving Fisher (Wiley, New York) 231-256.

U.S. Department of Health and Human Services, Social Security Administration, 1981, Social security bulletin, Annual statistical supplement (U.S. Government Printing Office, Washington, DC).

U.S. Department of Labor, Bureau of Labor Statistics, 1980, Employment and earnings 27 (U.S. Government Printing Office, Washington, DC).

Weber, W.E., 1975, Interest rates, inflation, and consumer expenditures, American Economic Review 65, 843-858.

Yaari, M., 1965, Uncertain lifetime, life insurance, and the theory of the consumer, Review of Economic Studies 32, 137-150. 